\title{
PERSISTENCE PROPERTIES AND UNIQUE CONTINUATION OF SOLUTIONS OF THE CAMASSA-HOLM EQUATION
}

\author{
A. ALEXANDROU HIMONAS, GERARD MISIOŁEK, GUSTAVO PONCE, AND YONG ZHOU
}

\begin{abstract}
It is shown that a strong solution of the Camassa-Holm equation, initially decaying exponentially together with its spacial derivative, must be identically equal to zero if it also decays exponentially at a later time. In particular, a strong solution of the Cauchy problem with compact initial profile can not be compactly supported at any later time unless it is the zero solution.
\end{abstract}

\section{INTRODUCTION}

This work is concerned with the nonperiodic Camassa-Holm equation

$$
\partial_{t} u-\partial_{t} \partial_{x}^{2} u+3 u \partial_{x} u-2 \partial_{x} u \partial_{x}^{2} u-u \partial_{x}^{3} u=0, \quad t, x \in \mathbb{R} .
$$

This equation appears in the context of hereditary symmetries studied by Fuchssteiner and Fokas [FF. It was first written explicitly, and derived physically as a water wave equation by Camassa and Holm [CH], who also studied its solutions. Equation (1.1) is remarkable for its properties such as infinitely many conserved integrals, bi-hamiltonian structure or its non-smooth travelling wave solutions known as "peakons" (see formula (1.9)). It was also derived as an equation for geodesics of the $H^{1}$-metric on the diffeomorphism group, see [Mi]. For a discussion of how it relates to the theory of hereditary symmetries see [F]. The inverse scattering approach to the Camassa-Holm equation has also been developed in several works, for example see [CH], $\mathrm{CoMc}$, [Mc], BSS], and references therein.

A considerable amount of work has been devoted to the study of the corresponding Cauchy problem in both nonperiodic and periodic cases. Among these results, of relevance to the present paper will be the fact that (1.1) is locally well-posed (in Hadamard's sense) in $H^{s}(\mathbb{R})$ for any $s>3 / 2$, see for example $[\mathrm{LO},[\mathrm{R},[\mathrm{D}$. The long time behaviour of solutions has been studied and conditions which guarantee their global existence and their finite blow up have been deduced. In particular, in [Mc] a necessary and sufficient condition was established on the initial datum to guarantee finite time singularity formation for the corresponding strong solution. For further results in this direction we refer to $\underline{\mathrm{Mc}}, \mathrm{CoE}]$ and the survey article $\mathrm{MO}$ and references therein. For well-posedness results in the periodic case we refer to [HM1], [Mi], and [DKT], where the equation is studied in its integral-differential form (see (1.2) below) as an ODE on the space of diffeomorphisms of the circle. A recent result demonstrating that the solution map $u_{0} \rightarrow u$ for 
the Camassa-Holm equation is not locally uniformly continuous in Sobolev spaces can be found in HM2.

Also the Camassa-Holm equation has been studied as an integrable infinite-dimensional Hamiltonian system, and several works have been devoted to several aspect of its scattering setting, see [CH], $\mathrm{CoMc}, \mathrm{Mc}, \mathrm{BSS}]$ and references therein.

It is convenient to rewrite the equation in its formally equivalent integral-differential form

$$
\partial_{t} u+u \partial_{x} u+\partial_{x} G *\left(u^{2}+\frac{1}{2}\left(\partial_{x} u\right)^{2}\right)=0,
$$

where $G(x)=e^{-|x|}$.

Our first objective here is to formulate decay conditions on a solution, at two distinct times, which guarantee that $u \equiv 0$ is the unique solution of equation (1.1). The idea of proving unique continuation results for nonlinear dispersive equations under decay assumptions of the solution at two different times was motivated by the recent works EKPV1], EKPV2 on the nonlinear Schrödinger and the $k$-generalized Korteweg-de Vries equations respectively.

In the recent works $[\mathrm{Co},[\mathrm{He}$ and $[\mathrm{Z}]$ it was shown that $u$ cannot preserve compact support in a non-trivial time interval (i.e. for $t \in[0, \epsilon], \epsilon>0$ ) except if $u \equiv 0$. However, this result does not preclude the possibility of the solution having compact support at a later time. In fact, in [Z the question concerning the possibility of a smooth solution of (1.1) having compact support at two different times was explicitly stated. In particular, our first result, Theorem 1.1. gives a negative answer to this question.

Theorem 1.1. Assume that for some $T>0$ and $s>3 / 2$

$$
u \in C\left([0, T]: H^{s}(\mathbb{R})\right)
$$

is a strong solution of the IVP associated to the equation (1.2). If $u_{0}(x)=u(x, 0)$ satisfies that for some $\alpha \in(1 / 2,1)$

$$
\left|u_{0}(x)\right| \sim o\left(e^{-x}\right), \quad \text { and } \quad\left|\partial_{x} u_{0}(x)\right| \sim O\left(e^{-\alpha x}\right) \quad \text { as } x \uparrow \infty,
$$

and there exists $t_{1} \in(0, T]$ such that

$$
\left|u\left(x, t_{1}\right)\right| \sim o\left(e^{-x}\right) \quad \text { as } \quad x \uparrow \infty,
$$

then $u \equiv 0$.

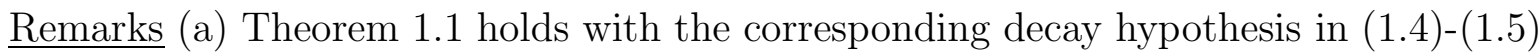
stated for $x<0$.

(b) The time interval $[0, T]$ is the maximal existence time interval of the strong solution. This guarantees that the solution is uniformly bounded in the $H^{s}$-norm in this interval (see (2.12) ), and that our solution is the strong limit of smooth ones such that the integration by parts in the proof (see (2.21), (2.29) ) can be justified. 
The proof of Theorem 1.1 will be a consequence of the following result concerning some persistence properties of the solution of the equation (1.2) in $L^{\infty}$-spaces with exponential weights.

Theorem 1.2. Assume that for some $T>0$ and $s>3 / 2$

$$
u \in C\left([0, T]: H^{s}(\mathbb{R})\right)
$$

is a strong solution of the IVP associated to the equation (1.2) and that $u_{0}(x)=u(x, 0)$ satisfies that for some $\theta \in(0,1)$

$$
\left|u_{0}(x)\right|, \quad\left|\partial_{x} u_{0}(x)\right| \sim O\left(e^{-\theta x}\right) \quad \text { as } \quad x \uparrow \infty .
$$

Then

$$
|u(x, t)|, \quad\left|\partial_{x} u(x, t)\right| \sim O\left(e^{-\theta x}\right) \text { as } \quad x \uparrow \infty,
$$

uniformly in the time interval $[0, T]$.

The following result establishes the optimality of Theorem 1.1 and tells us that a strong non-trivial solution of (1.2) corresponding to data with fast decay at infinity will immediately behave asymptotically, in the $x$-variable at infinity, as the "peakon" solution

$$
v_{c}(x, t)=c e^{-|x-c t|}, \quad t>0 .
$$

Theorem 1.3. Assume that for some $T>0$ and $s>3 / 2$

$$
u \in C\left([0, T]: H^{s}(\mathbb{R})\right)
$$

is a strong solution of the IVP associated to the equation (1.2) and that $u_{0}(x)=u(x, 0)$ satisfies that for some $\alpha \in(1 / 2,1)$

$$
\left|u_{0}(x)\right| \sim O\left(e^{-x}\right), \quad\left|\partial_{x} u_{0}(x)\right| \sim O\left(e^{-\alpha x}\right) \text { as } x \uparrow \infty
$$

for some $\alpha \in(1 / 2,1)$. Then

$$
|u(x, t)| \sim O\left(e^{-x}\right) \text { as } x \uparrow \infty,
$$

uniformly in the time interval $[0, T]$.

In the case when the solution $u(x, t)$ possesses further regularity and its data $u_{0}$ has stronger decay properties we shall give a more precise description of its behavior at infinity in the space variable.

As it was noted in both [Co and [Z] in the case of compactly supported initial data $u_{0}$ the difference $h(x, t)$ of the solution and its second derivative, i.e.

$$
h(x, t)=\left(1-\partial_{x}^{2}\right) u(x, t),
$$

remains compactly supported. Thus, if $u_{0}$ is supported in the interval $[a, b]$ in its lifespan, one has that $h(x, t)$ has compact support in the time interval $[\eta(a, t), \eta(b, t)]$, (for the definition of $\eta(\cdot, \cdot)$ see (2.38) $)$. 
Theorem 1.4. Assume that for some $T>0$ and $s>5 / 2$

$$
u \in C\left([0, T]: H^{s}(\mathbb{R})\right)
$$

is a strong solution of the IVP associated to the equation (1.2).

(a) If $u_{0}(x)=u(x, 0)$ has compact support, then for any $t \in(0, T]$

$$
u(x, t)=\left\{\begin{array}{lll}
c_{+}(t) e^{-x}, & \text { for } & x>\eta(b, t), \\
c_{-}(t) e^{x}, & \text { for } & x<\eta(a, t) .
\end{array}\right.
$$

(b) If for some $\mu>0$

$$
\partial_{x}^{j} u_{0} \sim O\left(e^{-(1+\mu)|x|}\right) \quad \text { as } \quad|x| \uparrow \infty \quad j=0,1,2,
$$

then for any $t \in(0, T]$

$$
h(x, t)=\left(1-\partial_{x}^{2}\right) u(x, t) \sim O\left(e^{-(1+\mu)|x|}\right) \quad \text { as } \quad|x| \uparrow \infty,
$$

and

$$
\lim _{x \rightarrow \pm \infty} e^{ \pm x} u(x, t)=c_{ \pm}(t)
$$

where in (1.15), (1.18) $c_{+}(\cdot), c_{-}(\cdot)$ denote continuous non-vanishing functions, with $c_{+}(t)>0$ and $c_{-}(t)<0$ for $t \in(0, T]$. Furthemore, $c_{+}(\cdot)$ is strictly increasing function, while $c_{-}(\cdot)$ is strictly decreasing.

Theorem 1.4 tells us that, as long as it exists, the solution $u(x, t)$ is positive at infinity and negative at minus infinity regardless of the profile of a fast-decaying data $u_{0} \neq 0$.

\section{Proof of the RESUlts}

First, assuming the result in Theorem [1.2 we shall prove Theorem 1.1.

Proof of Theorem 1.1. Integrating equation (1.2) over the time interval $\left[0, t_{1}\right]$ we get

$$
u\left(x, t_{1}\right)-u(x, 0)+\int_{0}^{t_{1}} u \partial_{x} u(x, \tau) d \tau+\int_{0}^{t_{1}} \partial_{x} G *\left(u^{2}+\frac{1}{2}\left(\partial_{x} u\right)^{2}\right)(x, \tau) d \tau=0 .
$$

By hypothesis (1.4) and (1.5) we have

$$
u\left(x, t_{1}\right)-u(x, 0) \sim o\left(e^{-x}\right) \text { as } x \uparrow \infty .
$$

From (1.4) and Theorem 1.2 it follows that

$$
\int_{0}^{t_{1}} u \partial_{x} u(x, \tau) d \tau \sim O\left(e^{-2 \alpha x}\right) \quad \text { as } x \uparrow \infty
$$

and so

$$
\int_{0}^{t_{1}} u \partial_{x} u(x, \tau) d \tau \sim o\left(e^{-x}\right) \quad \text { as } x \uparrow \infty
$$


We shall show that if $u \neq 0$, then the last term in (2.1) is $O\left(e^{-x}\right)$ but not $o\left(e^{-x}\right)$. Thus, we have

$$
\begin{aligned}
\int_{0}^{t_{1}} \partial_{x} G *\left(u^{2}+\frac{1}{2}\left(\partial_{x} u\right)^{2}\right)(x, \tau) d \tau & =\partial_{x} G * \int_{0}^{t_{1}}\left(u^{2}+\frac{1}{2}\left(\partial_{x} u\right)^{2}\right)(x, \tau) d \tau \\
& =\partial_{x} G * \rho(x),
\end{aligned}
$$

where by (1.4) and Theorem 1.2

$$
0 \leq \rho(x) \sim O\left(e^{-2 \alpha x}\right), \quad \text { so that } \quad \rho(x) \sim o\left(e^{-x}\right) \text { as } x \uparrow \infty .
$$

Therefore

$$
\begin{aligned}
\partial_{x} G * \rho(x) & =-\int_{-\infty}^{\infty} \operatorname{sgn}(x-y) e^{-|x-y|} \rho(y) d y \\
& =-e^{-x} \int_{-\infty}^{x} e^{y} \rho(y) d y+e^{x} \int_{x}^{\infty} e^{-y} \rho(y) d y .
\end{aligned}
$$

From (2.6) it follows that

$$
e^{x} \int_{x}^{\infty} e^{-y} \rho(y) d y=o(1) e^{x} \int_{x}^{\infty} e^{-2 y} d y \sim o(1) e^{-x} \sim o\left(e^{-x}\right),
$$

and if $\rho \neq 0$ one has that

$$
\int_{-\infty}^{x} e^{y} \rho(y) d y \geq c_{0}, \quad \text { for } \quad x \gg 1
$$

Hence, the last term in (2.5) and (2.7) satisfies

$$
-\partial_{x} G * \rho(x) \geq \frac{c_{0}}{2} e^{-x}, \quad \text { for } \quad x \gg 1
$$

which combined with (2.1)-(2.3) yields a contradiction. Thus, $\rho(x) \equiv 0$ and consequently $u \equiv 0$, see (2.5) .

Proof of Theorem 1.3. This proof is similar to that given for Theorem 1.1 and therefore it will be omitted.

We proceed to prove Theorem 1.2

Proof of Theorem 1.2. We introduce the following notations

$$
F(u)=u^{2}+\frac{\left(\partial_{x} u\right)^{2}}{2}
$$

and

$$
M=\sup _{t \in[0, T]}\|u(t)\|_{H^{s}} .
$$


Multiplying the equation (1.2) by $u^{2 p-1}$ with $p \in \mathbb{Z}^{+}$and integrating the result in the $x$-variable one gets

$$
\int_{-\infty}^{\infty} u^{2 p-1} \partial_{t} u d x+\int_{-\infty}^{\infty} u^{2 p-1} u \partial_{x} u d x+\int_{-\infty}^{\infty} u^{2 p-1} \partial_{x} G * F(u) d x=0 .
$$

The estimates

$$
\int_{-\infty}^{\infty} u^{2 p-1} \partial_{t} u d x=\frac{1}{2 p} \frac{d}{d t}\|u(t)\|_{2 p}^{2 p}=\|u(t)\|_{2 p}^{2 p-1} \frac{d}{d t}\|u(t)\|_{2 p}
$$

and

$$
\left|\int_{-\infty}^{\infty} u^{2 p-1} u \partial_{x} u d x\right| \leq\left\|\partial_{x} u(t)\right\|_{\infty}\|u(t)\|_{2 p}^{2 p}
$$

and Hölder's inequality in (2.13) yield

$$
\frac{d}{d t}\|u(t)\|_{2 p} \leq\left\|\partial_{x} u(t)\right\|_{\infty}\|u(t)\|_{2 p}+\left\|\partial_{x} G * F(u)(t)\right\|_{2 p}
$$

and therefore, by Gronwall's inequality

$$
\|u(t)\|_{2 p} \leq\left(\|u(0)\|_{2 p}+\left\|\partial_{x} G * F(u)(\tau)\right\|_{2 p} d \tau\right) e^{M t} .
$$

Since $f \in L^{2}(\mathbb{R}) \cap L^{\infty}(\mathbb{R})$ implies

$$
\lim _{q \uparrow \infty}\|f\|_{q}=\|f\|_{\infty}
$$

taking the limits in (2.17) (notice that $\partial_{x} G \in L^{1}$ and $F(u) \in L^{1} \cap L^{\infty}$ ) from (2.18) we get

$$
\|u(t)\|_{\infty} \leq\left(\|u(0)\|_{\infty}+\int_{0}^{t}\left\|\partial_{x} G * F(u)(\tau)\right\|_{\infty} d \tau\right) e^{M t} .
$$

Next, differentiating (1.2) in the $x$-variable produces the equation

$$
\partial_{t} \partial_{x} u+u \partial_{x}^{2} u+\left(\partial_{x} u\right)^{2}+\partial_{x}^{2} G *\left(u^{2}+\frac{1}{2}\left(\partial_{x} u\right)^{2}\right)=0 .
$$

Again, multiplying the equation (2.20) by $\partial_{x} u^{2 p-1}\left(p \in \mathbb{Z}^{+}\right)$integrating the result in the $x$-variable and using integration by parts

$$
\int_{-\infty}^{\infty} u \partial_{x}^{2} u\left(\partial_{x} u\right)^{2 p-1} d x=\int_{-\infty}^{\infty} u \partial_{x}\left(\frac{\left(\partial_{x} u\right)^{2 p}}{2 p}\right) d x=-\frac{1}{2 p} \int_{-\infty}^{\infty} \partial_{x} u\left(\partial_{x} u\right)^{2 p} d x
$$

one gets the inequality

$$
\frac{d}{d t}\left\|\partial_{x} u(t)\right\|_{2 p} \leq 2\left\|\partial_{x} u(t)\right\|_{\infty}\left\|\partial_{x} u(t)\right\|_{2 p}+\left\|\partial_{x}^{2} G * F(u)(t)\right\|_{2 p}
$$

and therefore as before

$$
\left\|\partial_{x} u(t)\right\|_{2 p} \leq\left(\left\|\partial_{x} u(0)\right\|_{2 p}+\int_{0}^{t}\left\|\partial_{x}^{2} G * F(u)(\tau)\right\|_{2 p} d \tau\right) e^{2 M t}
$$


Since $\partial_{x}^{2} G=G-\delta$, we can use (2.18) and pass to the limit in (2.23) to obtain

$$
\left\|\partial_{x} u(t)\right\|_{\infty} \leq\left(\left\|\partial_{x} u(0)\right\|_{\infty}+\int_{0}^{t}\left\|\partial_{x}^{2} G * F(u)(\tau)\right\|_{\infty} d \tau\right) e^{2 M t} .
$$

We shall now repeat the above arguments using the weight

$$
\varphi_{N}(x)= \begin{cases}1, & x \leq 0, \\ e^{\theta x}, & x \in(0, N), \\ e^{\theta N}, & x \geq N\end{cases}
$$

where $N \in \mathbb{Z}^{+}$. Observe that for all $N$ we have

$$
0 \leq \varphi_{N}^{\prime}(x) \leq \varphi_{N}(x) \quad \text { a.e. } \quad x \in \mathbb{R} .
$$

Using notation in (2.11), from equation (1.2) we obtain

$$
\partial_{t}\left(u \varphi_{N}\right)+\left(u \varphi_{N}\right) \partial_{x} u+\varphi_{N} \partial_{x} G * F(u)=0,
$$

while from (2.20) we get

$$
\partial_{t}\left(\partial_{x} u \varphi_{N}\right)+u \partial_{x}^{2} u \varphi_{N}+\left(\partial_{x} u \varphi_{N}\right) \partial_{x} u+\varphi_{N} \partial_{x}^{2} G * F(u)=0,
$$

We need to eliminate the second derivatives in the second term in (2.28). Thus, combining integration by parts and (2.26) we find

$$
\begin{aligned}
\left|\int_{-\infty}^{\infty} u \partial_{x}^{2} u \varphi_{N}(x)\left(\partial_{x} u \varphi_{N}(x)\right)^{2 p-1} d x\right| \\
\quad=\left|\int_{-\infty}^{\infty} u\left(\partial_{x} u \varphi_{N}(x)\right)^{2 p-1}\left(\partial_{x}\left(\partial_{x} u \varphi_{N}(x)\right)-\partial_{x} u \varphi_{N}^{\prime}(x)\right) d x\right| \\
\quad=\left|\int_{-\infty}^{\infty} u \partial_{x}\left(\frac{\left(\partial_{x} u \varphi_{N}(x)\right)^{2 p}}{2 p}\right) d x-\int_{-\infty}^{\infty} u \partial_{x} u \varphi_{N}^{\prime}(x)\left(\partial_{x} u \varphi_{N}(x)\right)^{2 p-1} d x\right| \\
\leq 2\left(\|u(t)\|_{\infty}+\left\|\partial_{x} u(t)\right\|_{\infty}\right)\left\|\partial_{x} u \varphi_{N}\right\|_{2 p}^{2 p}
\end{aligned}
$$

Hence, as in the weightless case (2.19) and (2.24), we get

$$
\begin{aligned}
\left\|u(t) \varphi_{N}\right\|_{\infty} & +\left\|\partial_{x} u(t) \varphi_{N}\right\|_{\infty} \leq e^{2 M t}\left(\left\|u(0) \varphi_{N}\right\|_{\infty}+\left\|\partial_{x} u(0) \varphi_{N}\right\|_{\infty}\right) \\
& +e^{2 M t} \int_{0}^{t}\left(\left\|\varphi_{N} \partial_{x} G * F(u)(\tau)\right\|_{\infty}+\left\|\varphi_{N} \partial_{x}^{2} G * F(u)(\tau)\right\|_{\infty}\right) d \tau .
\end{aligned}
$$

A simple calculation shows that there exists $c_{0}>0$, depending only on $\theta \in(0,1)$ (see (1.7) and (2.25) ) such that for any $N \in \mathbb{Z}^{+}$

$$
\varphi_{N}(x) \int_{-\infty}^{\infty} e^{-|x-y|} \frac{1}{\varphi_{N}(y)} d y \leq c_{0} .
$$


Thus, for any appropriate function $f$ one sees that

$$
\begin{aligned}
\left|\varphi_{N} \partial_{x} G * f^{2}(x)\right| & =\left|\varphi_{N}(x) \int_{-\infty}^{\infty} \operatorname{sgn}(x-y) e^{-|x-y|} f^{2}(y) d y\right| \\
& \leq \varphi_{N}(x) \int_{-\infty}^{\infty} e^{-|x-y|} \frac{1}{\varphi_{N}(y)} \varphi_{N}(y) f(y) f(y) d y \\
& \leq\left(\varphi_{N}(x) \int_{-\infty}^{\infty} e^{-|x-y|} \frac{1}{\varphi_{N}(y)} d y\right)\left\|\varphi_{N} f\right\|_{\infty}\|f\|_{\infty} \\
& \leq c_{0}\left\|\varphi_{N} f\right\|_{\infty}\|f\|_{\infty} .
\end{aligned}
$$

Since $\partial_{x}^{2} G=G-\delta$ the argument in (2.32) also shows that

$$
\left|\varphi_{N} \partial_{x}^{2} G * f^{2}(x)\right| \leq c_{0}\left\|\varphi_{N} f\right\|_{\infty}\|f\|_{\infty}
$$

Thus, inserting (2.32)-(2.33) into (2.30) and using (2.11)-(2.12) it follows that there exits a constant $\tilde{c}=\tilde{c}(M ; T)>0$ such that

$$
\begin{aligned}
& \left\|u(t) \varphi_{N}\right\|_{\infty}+\left\|\partial_{x} u(t) \varphi_{N}\right\|_{\infty} \leq \tilde{c}\left(\left\|u(0) \varphi_{N}\right\|_{\infty}+\left\|\partial_{x} u(0) \varphi_{N}\right\|_{\infty}\right) \\
& \quad+\tilde{c} \int_{0}^{t}\left(\|u(\tau)\|_{\infty}+\left\|\partial_{x} u(\tau)\right\|_{\infty}\right)\left(\left\|u(\tau) \varphi_{N}\right\|_{\infty}+\left\|\partial_{x} u(\tau) \varphi_{N}\right\|_{\infty}\right) d \tau \\
& \quad \leq \tilde{c}\left(\left\|u(0) \varphi_{N}\right\|_{\infty}+\left\|\partial_{x} u(0) \varphi_{N}\right\|_{\infty}+\int_{0}^{t}\left(\left\|u(\tau) \varphi_{N}\right\|_{\infty}+\left\|\partial_{x} u(\tau) \varphi_{N}\right\|_{\infty}\right) d \tau\right) .
\end{aligned}
$$

Hence, for any $N \in \mathbb{Z}^{+}$and any $t \in[0, T]$ we have

$$
\begin{aligned}
\left\|u(t) \varphi_{N}\right\|_{\infty}+\left\|\partial_{x} u(t) \varphi_{N}\right\|_{\infty} & \leq \tilde{c}\left(\left\|u(0) \varphi_{N}\right\|_{\infty}+\left\|\partial_{x} u(0) \varphi_{N}\right\|_{\infty}\right) \\
& \leq \tilde{c}\left(\left\|u(0) e^{\theta x}\right\|_{\infty}+\left\|\partial_{x} u(0) e^{\theta x}\right\|_{\infty}\right) .
\end{aligned}
$$

Finally, taking the limit as $N$ goes to infinity in (2.35) we find that for any $N \in \mathbb{Z}^{+}$and any $t \in[0, T]$

$$
\sup _{t \in[0, T]}\left(\left\|u(t) e^{\theta x}\right\|_{\infty}+\left\|\partial_{x} u(t) e^{\theta x}\right\|_{\infty}\right) \leq \tilde{c}\left(\left\|u(0) e^{\theta x}\right\|_{\infty}+\left\|\partial_{x} u(0) e^{\theta x}\right\|_{\infty}\right),
$$

which completes the proof of Theorem 2 .

It remains to prove Theorem 1.4 .

Proof of Theorem 1.4. A simple calculation shows that the solution $u$ of equation (1.1) satisfies the identity

$$
\left(1-\partial_{x}^{2}\right) u \circ \eta\left(\partial_{x} \eta\right)^{2}=\left(1-\partial_{x}^{2}\right) u_{0}
$$


(it has a mechanical interpretation as conservation of spacial angular momentum). Here $\eta=\eta(x, t)$ is the flow of $u$, that is

$$
\begin{cases}\frac{d \eta(x, t)}{d t} & =u(\eta(x, t), t) \\ \eta(x, 0) & =x\end{cases}
$$

so that by the assumption and the standard ODE theory $t \rightarrow \eta(t)$ is a smooth curve of $C^{1}$-diffeomorphisms of the line, close to the identity map and defined on the same time interval as $u$ (see Mi] for details in the periodic case). From (2.37) we then have

$$
u(x, t)=\int_{-\infty}^{\infty} e^{-|x-y|} h(y, t) d y=e^{-x} \int_{-\infty}^{x} e^{y} h(y, t) d y+e^{x} \int_{x}^{\infty} e^{-y} h(y, t) d y,
$$

where

$$
h(x, t)=\left(1-\partial_{x}^{2}\right) u(x, t)=\frac{\left(1-\partial_{x}^{2}\right) u_{0}\left(\eta^{-1}(x, t)\right)}{\left(\partial_{x} \eta\left(\eta^{-1}(x, t), t\right)\right)^{2}} .
$$

Let us first prove part (a).

Thus, from (2.40) it follows that if $u_{0}$ has compact support in $x$ in the interval $[a, b]$, then so does $h(\cdot, t)$ in nthe interval $[\eta(a, t), \eta(b, t)]$, for any $t \in[0, T]$. Moreover, defining

$$
E_{+}(t)=\int_{\eta(a, t)}^{\eta(b, t)} e^{y} h(y, t) d y \quad \text { and } \quad E_{-}(t)=\int_{\eta(a, t)}^{\eta(b, t)} e^{-y} h(y, t) d y
$$

one has from (2.40) that

$$
u(x, t)=e^{-|x|} * h(x, t)=e^{-x} E_{+}(t), \quad x>\eta(b, t),
$$

and

$$
u(x, t)=e^{-|x|} * h(x, t)=e^{x} E_{-}(t), \quad x<\eta(a, t) .
$$

Hence, it follows that for $x>\eta(b, t)$

$$
u(x, t)=-\partial_{x} u(x, t)=\partial_{x}^{2} u(x, t)=e^{-x} E_{+}(t),
$$

and for $x<\eta(a, t)$

$$
u(x, t)=\partial_{x} u(x, t)=\partial_{x}^{2} u(x, t)=e^{x} E_{-}(t) .
$$

Next, integration by part, (2.44), (2.45), and the equation in (1.1) yield the identities

$$
\begin{aligned}
E_{+}(0) & =\int_{-\infty}^{\infty} e^{y} h(y, 0) d y=\int_{-\infty}^{\infty} e^{y} u_{0}(y) d y-\int_{-\infty}^{\infty} e^{y} \partial_{x}^{2} u_{0}(y) d y \\
& =\int_{-\infty}^{\infty} e^{y} u_{0}(y) d y+\int_{-\infty}^{\infty} e^{y} \partial_{x} u_{0}(y) d y=0
\end{aligned}
$$


and

$$
\begin{aligned}
& \frac{d E_{+}(t)}{d t} \\
& =-\int_{-\infty}^{\infty} e^{y} u \partial_{x} u d y+\int_{-\infty}^{\infty} e^{y} \partial_{x}^{2}\left(u \partial_{x} u\right) d y-\int_{-\infty}^{\infty} e^{y} \partial_{x} F(u) d y \\
& =\left.e^{y}\left(\partial_{x}\left(u \partial_{x} u\right)-u \partial_{x} u\right)\right|_{-\infty} ^{\infty}-\left.e^{y}\left(u^{2}+\frac{\left(\partial_{x} u\right)^{2}}{2}\right)\right|_{-\infty} ^{\infty}+\int_{-\infty}^{\infty} e^{y} F(u) d y \\
& =\int_{-\infty}^{\infty} e^{y}\left(u^{2}+\frac{\left(\partial_{x} u\right)^{2}}{2}\right)(y, t) d y>0 .
\end{aligned}
$$

Therefore, in the life-span of the solution $u(x, t), E_{+}(t)$ is an increasing function. Thus, from (2.46) it follows that $E_{+}(t)>0$ for $t \in(0, T]$.

Similarly, it is easy to see that $E_{-}(t)$ is decreasing with $E_{-}(0)=0$, therefore $E_{-}(t)<0$ for $t \in(0, T]$.

Taking $c_{ \pm}(t)=E_{ \pm}(t)$ we obtain (1.15).

Next, let us consider part (b). Since $h(x, t)=\left(1-\partial_{x}^{2}\right) u(x, t)$ satisfies the equation

$$
\partial_{t} h+u(x, t) \partial_{x} h=-2 \partial_{x} u(x, t) h, \quad(x, t) \in \mathbb{R} \times[0, T],
$$

an argument similar to that given in the proof of Theorem 1.2 shows that

$$
\sup _{t \in[0, T]}\left\|h(t) e^{(1+\mu)|x|}\right\|_{\infty} \leq \tilde{c}\left\|h(0) e^{(1+\mu)|x|}\right\|_{\infty},
$$

with $\tilde{c}$ depending only on $M$ in (2.12) and $T$, and that for any $\theta \in(0,1)$

$$
\partial_{x}^{j} u(t) \sim O\left(e^{-\theta|x|}\right) \quad \text { as } \quad|x| \uparrow \infty \quad \text { for } \quad j=0,1,2 .
$$

Thus, the definitions in (2.41) make sense with the integrals extended to the whole real line and the computations in (2.46)-(2.47) can be carried out in the same fashion. Finally, using (2.49) in (2.39) we obtain (1.18).

\section{Acknowledgments}

The authors would like to thank Prof. D. Holm for useful comments concerning this work. G. P. was supported by an NSF grant. Y. Z. was supported by NSFC under grant no. 10501012 and Shanghai Rising-Star Program 05QMX1417.

\section{REFERENCES}

[BSS] Beals, R., Sattinger, D., and Szmigielski, J., Multipeakons and the classical moment problem, Adv. Math. 154 (2000), no. 2, pp. 229-257.

[CH] Camassa, R. and Holm, D., An integrable shallow water equation with peaked solutions, Phys. Rev. Lett. 71 (1993), pp. 1661-1664. 
[Co] Constantin, A., Finite propagation speed for the Camassa-Holm equation, J. Math. Phys. 46 (2005), no 2, pp. 4

[CoE] Constantin, A. and Escher, J., Global existence and blow-up for a shallow water equation, Ann. Scuola Norm. Sup. Pisa Cl. Sci. 26 (1998), no. 2, pp. 303-328.

[CoMc] Constantin, A. and McKean, H., A shallow water equation on the circle, Comm. Pure Appl. Math. 52 (1999), pp. 949-982.

[CoS] Constantin, A. and Strauss, W., Stability of peakons, Comm. Pure Appl. Math. 53 (2000), pp. 603-610.

[D] Danchin, R., A few remarks on the Camassa-Holm equation, Differential Integral Equations 14 (2001), pp. 953-988.

[DKT] De Lellis, C., Kappeler, T. and Topalov, P., Low-regularity solutions of the periodic CamassaHolm equation, pre-print.

[EKPV1] Escauriaza, L., Kenig, C. E., Ponce, G., and Vega, L., On unique continuation of solutions of Schrödinger equations, to appear in Comm. PDE

[EKPV2] Escauriaza, L., Kenig, C. E., Ponce, G., and Vega, L., On uniqueness properties of solutions of the $k$-generalized KdV equations, pre-print.

[FF] Fuchssteiner, B. and Fokas, A., Symplectic structures, their Backlund transformations and hereditary symmetries, Phys. D 4 (1981/1982), pp. 47-66.

[F] Fuchssteiner, B., Some tricks from the symmetry-toolbox for nonlinear equations: generalization of the Camassa-Holm equation, Physica D 95, (1996), pp. 229-243.

[He] Henry, D., Compactly supported solutions of the Camassa-Holm equation, J. Nonlinear Math. Phys., 12 (2005), pp. 342-347.

[HM1] Himonas, A. and Misiołek G., The Cauchy problem for an integrable shallow water equation, Differential Integral Equations 14 (2001), pp. 821-831.

[HM2] Himonas, A. and Misiołek G., High-frequency smooth solutions and well-posedness of the Camassa-Holm equation, Int. Math. Res. Not. 51 (2005), pp. 3135-3151.

[LO] Li, Y. and Olver, P., Well-posedness and blow-up solutions for an integrable nonlinearly dispersive model wave equation, J. Differential Equations 162 (2000), pp. 27-63.

[Mc] McKean, H., Breakdown of the Camassa-Holm equation, Comm. Pure Appl. Math. 57 (2004), pp. 416-418.

[Mi] Misiołek, G., Classical solutions of the periodic Camassa-Holm equation, Geom. Funct. Anal. 12 (2002), pp. 1080-1104.

[Mo] Molinet, L., On well-posedness results for the Camassa-Holm equation on the line: A survey, J. Nonlin. Math. Phys. 11 (2004), pp. 521-533.

[R] Rodriguez-Blanco, G., On the Cauchy problem for the Camassa-Holm equation, Nonlinear Anal. 46 (2001), pp. 309-327.

[Z] Zhou, Y., Infinite propagation speed for a shallow water equation, pre-print.

\section{A. Alexandrou Himonas \\ Department of Mathematics \\ University of Notre Dame \\ Notre Dame, IN 46556 \\ USA \\ E-mail: himonas.1@nd.edu}




\section{Gerard Misiołek}

Department of Mathematics

University of Notre Dame

Notre Dame, IN 46556

USA

E-mail: gmisiole@nd.edu

\section{Gustavo Ponce}

Department of Mathematics

University of California

Santa Barbara, CA 93106

USA

E-mail: gmisiole@nd.edu

\section{Yong Zhou}

Department of Mathematics

East China Normal University

Shangai 200062

China

E-mail: yzhou@math.ecnu.cn

and

Institute des Hautes Éudes Scientifiques

35 , route de Chartres

F-91440 Bures-sur-Yvette

France 